\title{
Instabilities in a horizontal liquid layer in cocurrent gas flow with an evaporating interface
}

\author{
R. Liu* \\ Key Laboratory of Microgravity (National Microgravity Laboratory), Institute of Mechanics, Chinese Academy of Sciences, Beijing 100190, \\ China and Chimie-Physique EP-CP165/62, Microgravity Research Center, Université Libre de Bruxelles, Avenue Roosevelt 50, \\ Bruxelles B-1050, Belgium \\ O. A. Kabov ${ }^{\dagger}$ \\ Chimie-Physique EP-CP165/62, Microgravity Research Center, Université Libre de Bruxelles, Avenue Roosevelt 50, Bruxelles B-1050, \\ Belgium Heat Transfer International Research Institute, Université Libre de Bruxelles, Brussels, Belgium Institute of Thermophysics, Russian \\ Academy of Sciences, Brussels, Belgium Institute of Thermophysics, Russian Academy of Sciences, Prospect Lavrentyev 1, Novosibirsk, \\ 630090, Russia and Centre of Smart Interfaces, Technische Universität Darmstadt, Petersenstrasse 30, Darmstadt 64287, Germany
}

(Received 26 December 2011; published 5 June 2012)

\begin{abstract}
The problem of a two-layer system consisting of a horizontal liquid layer in contact with its own vapor is considered. The liquid layer is bounded by a rigid wall from below, and phase change can occur at the interface. The flow of the vapor phase is driven by a constant pressure gradient in the streamwise direction. We have taken into account the effects of buoyancy, thermocapillarity, evaporation, and the dynamics of the vapor phase. A full linear stability analysis is performed using a Chebyshev spectral method. The influences of evaporation effect and the interfacial shear on the Rayleigh instability and the Marangoni instability have been studied. The results show that both the evaporation and the interfacial shear play important roles in the stability of the system.
\end{abstract}

DOI: 10.1103/PhysRevE.85.066305

PACS number(s): 47.20.Dr, 47.11.Kb

\section{INTRODUCTION}

Convection occurring in a horizontal liquid layer has received extensive attention since Bénard observed hexagonal roll cells upon the onset of convection in a layer of molten spermaceti with a free surface [1]. The phenomenon of convection in an evaporating liquid layer is of particular interest because of its importance in heat exchangers, distillation, drying technologies, and cooling of microelectronic equipment.

During evaporation, an essential mechanism is that evaporation leads to intensive cooling of the liquid-vapor interface. When the temperature drop induced by evaporation across the liquid layer exceeds a critical value, convective instability occurs. This instability mechanism has been studied by many previous investigators. Miller [2] examined the instability of an isothermal evaporating interface associated with a moving boundary. Prosperetti and Plesset [3] studied the stability of an evaporating liquid surface. In their analysis, the effect of viscosities of the vapor and the liquid is neglected, and the depths of the vapor and the liquid are infinite. Palmer investigated the hydrodynamic stability of rapidly evaporating liquids at reduced pressure in an infinite-depth liquid-vapor system [4]. Burelbach et al. investigated the nonlinear stability of evaporating and condensing liquid films [5]. Vapor recoil, thermocapillarity, and rupture instabilities are discussed in their papers.

Many past papers on evaporating liquid films were based on a one-sided model [5], and the vapor phase adjacent to the liquid layer was considered to be passive or infinitely deep. In this case, the dynamics of vapor was assumed to have little influence on the instability of the liquid layer, consequently, the dynamics of the vapor was neglected.

\footnotetext{
*liurong@imech.ac.cn

${ }^{\dagger}$ kabov@itp.nsc.ru
}

VanHook et al. have proposed a two-sided model to study long-wavelength surface-tension-driven Bénard convection in a nonevaporating liquid film in contact with a gas layer with a finite thickness [6]. Their results show that, if the ambient gas layer is thin enough, a two-sided model would better describe the system than a one-sided model. Ozen and Narayanan questioned the assumption that the vapor is passive in a vapor-liquid system with an evaporating interface [7]. They suggested that the active vapor layer plays an important role in the instability of the system. In order to take into account the effect of a finite-depth active vapor, they used a two-sided model to investigate the influence of evaporation on the instability. It should noted that, in their model, interfacial chemical potential equilibrium assumption is used, i.e., the temperature of the liquid is at its saturation value with respect to the vapor pressure.

The boundary conditions at the evaporating interface is complicated. A careful look at previous literature indicates that many sets of interfacial conditions have been used to describe the relation between the mass flux and other variables, such as the interfacial temperature, velocity, and pressure. Liu and Liu [8] gave up the assumption of interfacial chemical potential equilibrium and used a more general empirical relation, i.e., the Hertz-Knudsen equation [9], to describe the relation between the mass flux and the local temperature and the pressure of vapor. Kanatoni [10] has studied the interfacial instability induced by lateral vapor pressure fluctuation in a vapor-liquid system with an evaporating interface. More recently, Kanatani and Oron [11] have studied the nonlinear dynamics of the same system as that in Ref. [10]. In these two papers, the Hertz-Knudsen equation has also been applied to describe the dynamics of the interface. Margerit et al. [12] used a more general formulation taking into account the nonequilibrium effect contains a temperature discontinuity at the liquid-vapor interface during evaporation. However, the effect of this temperature jump may be neglected unless the phase change 
occurs too rapidly. More general formulations of dynamical sharp-interface conditions for phase transformations in viscous heat-conducting fluids have been investigated by Fried et al. [13] and Anderson et al. [14]. Shklyaev and Fried [15] used the new interfacial conditions proposed in Ref. [13] to revisit the problem of an evaporating thin liquid film.

In recent years, increasing performance demands in semiconductor technology, including shrinking feature size, increasing transistor density, and faster circuit speeds, have resulted in very high chip power dissipation and heat fluxes. It is also leading to greater nonuniformity of on-chip power dissipation, creating localized submillimeter hot spots, often exceeding $1 \mathrm{~kW} / \mathrm{cm}^{2}$ in heat flux, which can degrade the processor performance and reliability [16]. Similar developments are underway in microwave integrated circuits and power amplifier chips with even higher localized heat fluxes and heat densities. For more applications and related papers on these subjects, we refer the reader to the review paper by Kabov [17]. The industrial and technological applications mentioned above involve thin liquid films on uniform or nonuniform heated substrates. To avoid the reduction in their performance by film breakdown, it is of crucial importance to understand when and why instabilities arise that may result in the rupture of the film. Understanding the physical mechanisms of instability and rupture behavior is also highly desirable for the requirement of seeking effective ways to suppress the rupture of heated films.

Shear-induced flows of liquid films are important for a number of technological innovations for ground and space applications. A particularly promising technological candidate to prevent the rupture of film due to temperature gradients at the interface, which allows reaching high heat fluxes and minimizing space and mass of cooling equipment, is a setup where heat is transferred to a subcooled thin liquid film driven by a forced gas on one side of a minichannel. It is quite evident that the combined effects of evaporation, thermocapillarity, gas dynamics, and gravity as well as the formation of a microscopic adsorbed film on the wall, are somewhat complicated issues and have not yet been studied systematically. Recently, Gatapova and Kabov [18] have studied the problem of a locally heated liquid film sheared by gas flow in a channel. The problem of heat and mass transfer has been examined in the framework one-dimensional long-wave model. Iorio et al. [19] have studied evaporative convection in an open cavity under shear stress flow using direct numerical simulation. More recently, Kabov et al. [20] have studied experimentally the dynamics and evaporation of thin shear-driven liquid films in microgap channels. In the present paper, we investigate the instability of evaporating films driven by the action of vapor flow in a microchannel. The aim of the present paper is to provide physical insight into the mechanisms of instability of two phase flow in heated minichannels.

The present paper is organized as follows. In Sec. II, the mathematical formulation of the physical model is presented. We provide dimensionless equations and parameters in Sec. III. The numerical method is presented in Sec. IV. In Sec. V, we present the results and discussions. In Sec. VI, we summarize the results and present the conclusions.

\section{PROBLEM FORMULATION}

We consider a two-layer system consisting of a liquid layer of thickness $d_{l}$ underlying its own vapor of thickness $d_{v}$ as shown in Fig. 1. The system is infinite in the streamwise $(x)$ and the spanwise ( $y$ ) directions. The acceleration of gravity is opposite the $z$ direction. We assumed that the top wall and the bottom wall are rigid perfectly conducting boundaries. The gas flow is driven by a constant pressure gradient. The vapor and liquid phases are separated by an immiscible and deformable interface where phase change can occur. The surface tension $\sigma$ decreases linearly with temperature $T$, i.e., $\sigma=\sigma_{0}-\sigma_{T}(T-$ $T_{0}$ ), where $\sigma_{T}$ is a constant coefficient and $T_{0}$ is the temperature of the reference state.

The motions of the liquid and gas layers are governed by the continuity equation, the Navier-Stokes equation, and the energy equation for incompressible Newtonian fluids,

$$
\begin{gathered}
\nabla \cdot \boldsymbol{u}_{i}=0 \\
\partial_{t} \boldsymbol{u}_{i}+\boldsymbol{u}_{i} \cdot \nabla \boldsymbol{u}_{i}=-\frac{1}{\rho_{i}} \nabla p_{i}+\left[1-\beta_{i}\left(T_{i}-T_{0}\right)\right] \mathbf{g}+v_{i} \nabla^{2} \boldsymbol{u}_{i} \\
\partial_{t} T_{i}+\boldsymbol{u}_{i} \cdot \nabla T_{i}=\kappa_{i} \nabla^{2} T_{i}
\end{gathered}
$$

in which $\boldsymbol{u}, p$, and $T$ are the velocity, pressure, and temperature, respectively. $\rho, v$, and $\kappa$ are the density, kinematic viscosity, and thermal diffusivity. The subscript $i=v, l$ denotes the property of a vapor or liquid. For simplicity, we only consider the two-dimensional problem in the present paper.

We assume that, at the upper and the bottom walls, the isothermal condition for temperature and the no-slip condition for velocity are satisfied. So, at the bottom wall $\left(z=-d_{l}\right)$,

$$
T_{l}=T_{b}, \quad u_{l}=w_{l}=0,
$$

and, at the upper wall $\left(z=d_{v}\right)$,

$$
T_{v}=T_{u}, \quad u_{v}=w_{v}=0 .
$$

Here, $u$ and $w$ are the velocity components in the $x$ and $z$ directions, respectively.

The position of the interface can be described by $z=h(x, t)$. At the interface $z=h(x, t)$, a kinematic relation between $h(x, t)$ and the interfacial velocity $u_{\text {int }}$ is

$$
w_{\text {int }}=\partial_{t} h+u_{\text {int }} \partial_{x} h .
$$

$T u$

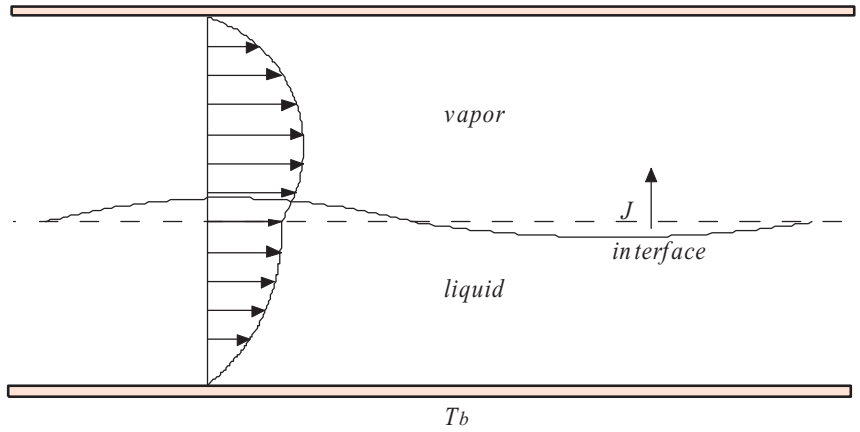

FIG. 1. (Color online) A sketch of the physical model. 
The mass balance equation at the interface is

$$
J=\rho_{v}\left(\boldsymbol{u}_{v}-u_{\text {int }}\right) \cdot \boldsymbol{n}=\left(\boldsymbol{u}_{l}-u_{\text {int }}\right) \cdot \boldsymbol{n},
$$

in which $J$ is the evaporation flux. The normal momentum and the tangential momentum balance conditions at the interface are

$$
\begin{gathered}
J[\boldsymbol{u}]_{v}^{l} \cdot \boldsymbol{n}+[p-\mathbf{P} \cdot \boldsymbol{n} \cdot \boldsymbol{n}]_{v}^{l}=-2 \sigma H, \\
{[\mathbf{P} \cdot \boldsymbol{n} \cdot \boldsymbol{t}]_{v}^{l}=\frac{\partial_{T} \sigma}{N}\left(\partial_{x} T+\partial_{x} h \partial_{z} T\right) .}
\end{gathered}
$$

Here, $[f]_{v}^{l}=f_{l}-f_{v}, \mathbf{P}$ is the viscous stress tensor, $\boldsymbol{n}$ and $\boldsymbol{t}$ are the unit vectors normal and tangent to the interface defined as

$$
\boldsymbol{n}=\frac{\left(-\partial_{x} h, 1\right)}{N}, \quad \boldsymbol{t}=\frac{\left(1, h_{x} \eta\right)}{N},
$$

in which $N=\sqrt{1+\left(\partial_{x} h\right)^{2}}, 2 H$ is the surface mean curvature defined as

$$
2 H=\frac{\partial_{x}^{2} h}{N^{3}} .
$$

The energy balance equation at the interface is

$$
J\left[\frac{1}{2}\left|\boldsymbol{u}-u_{\mathrm{int}}\right|^{2}\right]_{v}^{l}+q_{l}-q_{v}-\left[\mathbf{P} \cdot\left(\boldsymbol{u}-u_{\mathrm{int}}\right) \cdot \boldsymbol{n}\right]_{v}^{l}=J L,
$$

here, $L$ is the latent heat of evaporation and $q$ is the conductive heat flux defined as $-\chi \boldsymbol{n} \cdot \nabla T$ in which $\chi$ is the heat conductivity.

We assume that the temperatures of the liquid and vapor are continuous at the interface,

$$
T_{v}=T_{l} .
$$

The evaporation flux is given by the Hertz-Knudsen relation as

$$
J=\beta \sqrt{\frac{M}{2 \pi R T}}\left[p_{s}(T)-p_{v}\right],
$$

in which $\beta$ is the evaporation accommodation coefficient, $M$ is the molecular weight of vapor, $p_{s}(T)$ is the saturation pressure at temperature $T, p_{v}$ is the vapor pressure just beyond the interface, and $R$ is the universal gas constant.

The relation between the saturation pressure and the interfacial temperature is given by the Clausius-Clapeyron relation as

$$
p_{s}(T)=p_{0} \exp \left[-\frac{L}{R}\left(\frac{1}{T}-\frac{1}{T_{0}}\right)\right] .
$$

The tangential velocities of the liquid and the vapor layers are equal at the interface,

$$
\boldsymbol{u}_{v} \cdot \boldsymbol{t}=\boldsymbol{u}_{l} \cdot \boldsymbol{t}
$$

\section{DIMENSIONLESS EQUATIONS AND PARAMETERS}

In order to nondimensionalize the controlling equations, we scale length, time, velocity, pressure, temperature, and mass flux by $d_{l}, d_{l}^{2} / v_{l}, v_{l} / d_{l}, \rho_{l} v_{l}^{2} / d_{l}^{2}, \Delta T=T_{b}-T_{0}$, and $\chi_{l} \Delta T / d_{l} L$, respectively. The dimensionless equations are expressed as

$$
\begin{gathered}
\nabla \cdot \boldsymbol{u}_{l}=0 \\
\partial_{t} \boldsymbol{u}_{l}+\boldsymbol{u}_{l} \cdot \nabla \boldsymbol{u}_{l}=-\nabla p_{l}+\left(\operatorname{Pr}^{-1} \operatorname{Ra} \theta_{l}-G\right) \mathbf{e}_{z}+\nabla^{2} \boldsymbol{u}_{l} \\
\partial_{t} \theta_{l}+\boldsymbol{u}_{l} \cdot \nabla \theta_{l}=\operatorname{Pr}^{-1} \nabla^{2} \theta_{l} \\
\nabla \cdot \boldsymbol{u}_{v}=0 \\
\partial_{t} \boldsymbol{u}_{v}+\boldsymbol{u}_{v} \cdot \nabla \boldsymbol{u}_{v}=-\frac{1}{\rho^{*}} \nabla p_{v}+\left(\operatorname{Pr}^{-1} \beta^{*} \operatorname{Ra} \theta_{v}-G\right) \mathbf{e}_{z} \\
+v^{*} \nabla^{2} \boldsymbol{u}_{v} \\
\partial_{t} \theta_{v}+\boldsymbol{u}_{v} \cdot \nabla \theta_{v}=\operatorname{Pr}^{-1} \kappa^{*} \nabla^{2} \theta_{v} .
\end{gathered}
$$

Here, $\theta$ is defined as $\theta=\left(T-T_{0}\right) / \Delta T$. For convenience, we can choose $T_{0}=\bar{T}_{\text {int }}$.

The boundary conditions at the bottom wall $(z=-1)$ are

$$
u_{l}=w_{l}=0, \quad \theta_{l}=\left(T_{b}-T_{0}\right) /\left(T_{b}-\bar{T}_{\mathrm{int}}\right)=1 .
$$

The boundary condition at $z=d$,

$$
u_{v}=w_{v}=0, \quad \theta_{v}=\left(T_{u}-T_{0}\right) /\left(T_{b}-\bar{T}_{\mathrm{int}}\right)=-\frac{d}{\chi^{*}} .
$$

At the interface $z=h(x, t)$,

$$
\begin{aligned}
& E J=\rho^{*}\left(\boldsymbol{u}_{v}-u_{\mathrm{int}}\right) \cdot \boldsymbol{n}=\left(\boldsymbol{u}_{l}-u_{\mathrm{int}}\right) \cdot \boldsymbol{n}, \\
& u_{\text {int }} \cdot \boldsymbol{n}=\frac{\partial_{t} h}{N}, \\
& \boldsymbol{u}_{v} \cdot \boldsymbol{t}=\boldsymbol{u}_{l} \cdot \boldsymbol{t}, \\
& E J\left(\boldsymbol{u}_{l}-\boldsymbol{u}_{v}\right) \cdot \boldsymbol{n}+p_{l}-p_{v}-2 \boldsymbol{n} \cdot\left(\mathbf{E}_{l}-\mu^{*} \mathbf{E}_{v}\right) \cdot \boldsymbol{n} \\
& +2 S H=0 \text {, } \\
& \boldsymbol{n} \cdot\left(2 \mathbf{E}_{l}-2 \mu^{*} \mathbf{E}_{v}\right) \cdot \boldsymbol{t}=-\frac{\mathrm{Ma}}{\operatorname{Pr} N}\left(\partial_{x} \theta_{l}+\partial_{x} h \partial_{z} \theta_{l}\right), \\
& J-\frac{\Pi \Theta}{2 E} J\left\{\left|\boldsymbol{u}_{l}-u_{\text {int }}\right|^{2}-\left|\boldsymbol{u}_{v}-u_{\text {int }}\right|^{2}\right\}+\left(\nabla \theta_{l}-\chi^{*} \nabla \theta_{v}\right) \cdot \boldsymbol{n} \\
& +\frac{\Pi \Theta}{E^{2}} \boldsymbol{n} \cdot\left\{2 \mathbf{E}_{l} \cdot\left(\boldsymbol{u}_{l}-u_{\text {int }}\right)-2 \mu^{*} \mathbf{E}_{v} \cdot\left(\boldsymbol{u}_{v}-u_{\text {int }}\right)\right\} \\
& =0 \text {, } \\
& \theta_{l}=\theta_{v}, \\
& J=K\left(\theta_{l}-\frac{1}{\rho^{*}} \frac{\Pi}{E} p_{v}\right) .
\end{aligned}
$$

In these equations and boundary conditions, the parameters include

$$
\begin{aligned}
\mathrm{Ma} & =\frac{\sigma_{T} \Delta T d_{l}}{\mu_{l} \kappa_{l}}, \quad \mathrm{Ra}=\frac{\beta_{l} \rho_{l} g \Delta T d_{l}^{3}}{\mu_{l} \kappa_{l}}, \quad \text { Bo }=\frac{\beta_{l} \rho g d_{l}^{2}}{\sigma_{T}}, \\
G & =\frac{g \rho_{l}^{2} d_{l}^{3}}{\mu_{l}^{2}}, \quad \operatorname{Pr}=\frac{\nu_{l}}{\kappa_{l}}, \quad E=\frac{\chi_{l} \Delta T}{\mu_{l} L}, \quad S=\frac{\sigma \rho_{l} d_{l}}{\mu_{l}^{2}}, \\
\Pi & =\frac{\chi_{l} \mu_{l} T_{0}}{\left(\rho_{l} d_{l} L\right)^{2}}, \quad K=\beta \sqrt{\frac{M}{2 \pi R T_{0}}} \frac{\rho_{v} d_{l} L^{2}}{\chi_{l} T_{0}}, \quad \Theta=\frac{\Delta T}{T_{0}}, \\
\rho^{*} & =\frac{\rho_{v}}{\rho_{l}}, \quad \mu^{*}=\frac{\mu_{v}}{\mu_{l}}, \quad v^{*}=\frac{\nu_{v}}{\nu_{l}}, \\
\chi^{*} & =\frac{\chi_{v}}{\chi_{l}}, \quad \kappa^{*}=\frac{\kappa_{v}}{\kappa_{l}}, \quad d=\frac{d_{v}}{d_{l}} .
\end{aligned}
$$


(a)

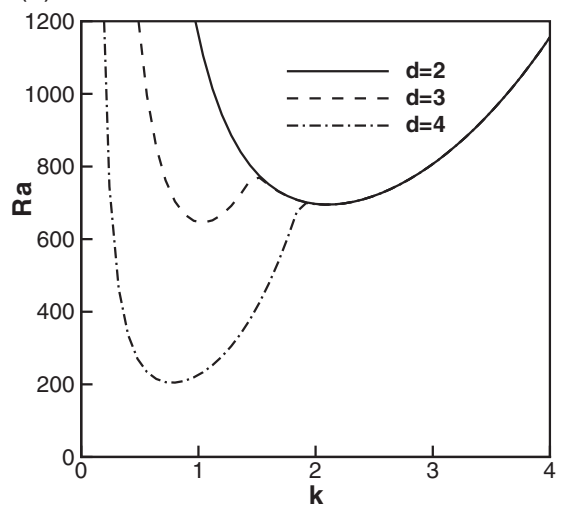

(b)

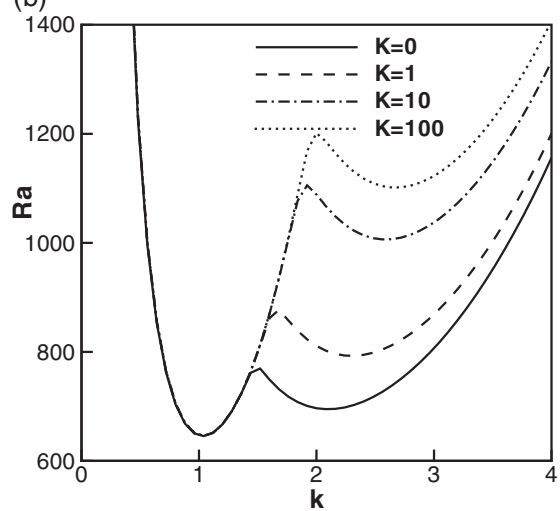

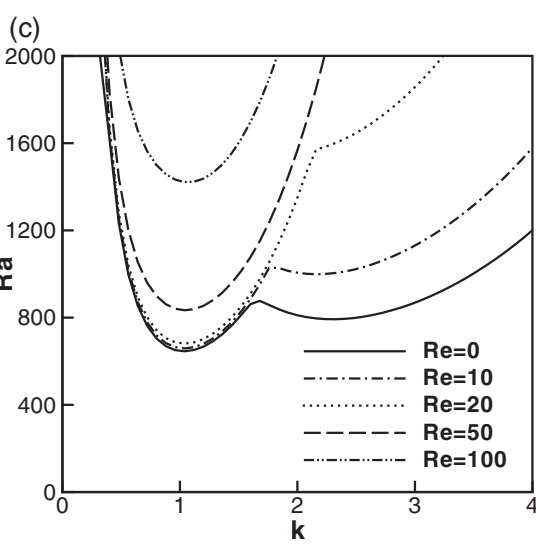

FIG. 2. (a) Effect of the depth ratio $d$ on the marginal curves of the critical Rayleigh number versus the wave number at $K=0$. (b) Effect of $K$ on the marginal curves of the critical Rayleigh number versus the wave number at $d=3$. (c) Effect of the Reynolds number Re on the marginal curves of the critical Rayleigh number versus the wave number at $d=3, K=1$.

\section{A. Basic state in dimensionless form}

In the basic state, we assume that the interface is flat, and the velocity and the temperature are independent of $x$. In this case, the applied pressure gradient in the vapor phase is the same as that in the liquid phase. The controlling equations for the streamwise velocity components are

$$
\begin{gathered}
-\partial_{x} \bar{p}+\mu^{*} \frac{\partial^{2} \bar{u}_{v}}{\partial z^{2}}=0, \\
-\partial_{x} \bar{p}+\frac{\partial^{2} \bar{u}_{l}}{\partial z^{2}}=0 .
\end{gathered}
$$

At the upper wall $z=d$,

$$
\bar{u}_{v}=0 .
$$

At the bottom wall $z=-1$,

$$
\bar{u}_{l}=0 \text {. }
$$

At the interface $z=0$,

$$
\bar{u}_{l}=\bar{u}_{v}, \quad \frac{\partial \bar{u}_{l}}{\partial z}=\mu^{*} \frac{\partial \bar{u}_{v}}{\partial z} .
$$

Solving the above equations, we have the basic velocities $\bar{u}_{v}(z)$ and $\bar{u}_{l}(z)$ in the form of

$$
\begin{aligned}
& \bar{u}_{v}=A_{1} z^{2}+B_{1} z+C_{1}, \\
& \bar{u}_{l}=A_{2} z^{2}+B_{2} z+C_{2} .
\end{aligned}
$$

The coefficients $A_{1}, B_{1}, C_{1}$ and $A_{2}, B_{2}, C_{2}$ are defined as

$$
\begin{gathered}
A_{1}=\frac{1}{2 \mu^{*}} \frac{\partial}{\partial x} \bar{p}, \quad B_{1}=\frac{1}{2} \frac{\mu^{*}-d^{2}}{\mu^{*}\left(d+\mu^{*}\right)} \frac{\partial}{\partial x} \bar{p}, \\
C_{1}=-\frac{1}{2} \frac{d(d+1)}{d+\mu^{*}} \frac{\partial}{\partial x} \bar{p}, \\
A_{2}=\frac{1}{2} \frac{\partial}{\partial x} \bar{p}, \quad B_{2}=\frac{1}{2} \frac{\mu^{*}-d^{2}}{d+\mu^{*}} \frac{\partial}{\partial x} \bar{p}, \\
C_{2}=-\frac{1}{2} \frac{d(d+1)}{d+\mu^{*}} \frac{\partial}{\partial x} \bar{p} .
\end{gathered}
$$

From the continuity equation and the boundary conditions of $\bar{v}_{v}(d)=0$ and $\bar{v}_{l}(-1)=0$, we can obtain the velocity components $\bar{v}_{v}(z)=0$ and $\bar{v}_{l}(z)=0$. In order to discuss the effect of interfacial shear on the stability of the problem, it is convenient to define the Reynolds number as $\operatorname{Re}=\frac{u_{\text {int }} d_{l}}{v_{l}}$ instead of $\frac{\partial}{\partial x} \bar{p}$. We note that the Reynolds number and the gradient of pressure are not independent. For the present problem, the Reynolds number $\operatorname{Re}=C_{2}$.

The unperturbed temperature fields satisfy

$$
\begin{aligned}
& \frac{\partial^{2} \bar{\theta}_{v}}{\partial z^{2}}=0, \\
& \frac{\partial^{2} \bar{\theta}_{l}}{\partial z^{2}}=0 .
\end{aligned}
$$

At the bottom wall $z=-1$,

$$
\bar{\theta}_{l}=1 \text {. }
$$

At the interface $z=0$,

$$
\bar{\theta}_{l}=\bar{\theta}_{v}=0, \quad \frac{\partial \bar{\theta}_{l}}{\partial z}=\chi^{*} \frac{\partial \bar{\theta}_{v}}{\partial z} .
$$

The basic temperatures $T_{v}(z)$ and $T_{l}(z)$ have the form

$$
\begin{aligned}
& \bar{\theta}_{v}=a_{1} z+b_{1}, \\
& \bar{\theta}_{l}=a_{2} z+b_{2} .
\end{aligned}
$$

Here,

$$
a_{1}=-\frac{1}{\chi^{*}}, \quad a_{2}=-1, \quad b_{1}=b_{2}=0 .
$$

In order to study the stability of infinitesimal disturbance on the basic state, we decompose the perturbations of velocity, pressure, and the temperature into normal modes,

$$
\left[u_{i}^{\prime}, w_{i}^{\prime}, p_{i}^{\prime}, \theta_{i}^{\prime}\right]=\left[U_{i}(z), W_{i}(z), P_{i}(z), \Theta_{i}(z)\right] \exp (\lambda t+i k x),
$$

in which $\lambda$ is the complex time growth rate and $k$ is the streamwise wave number. We denote $\frac{\partial}{\partial z}$ as " $D$ " and write the normal mode equations for the disturbances as follows:

For the vapor phase,

$$
i k U_{v}+D W_{v}=0
$$



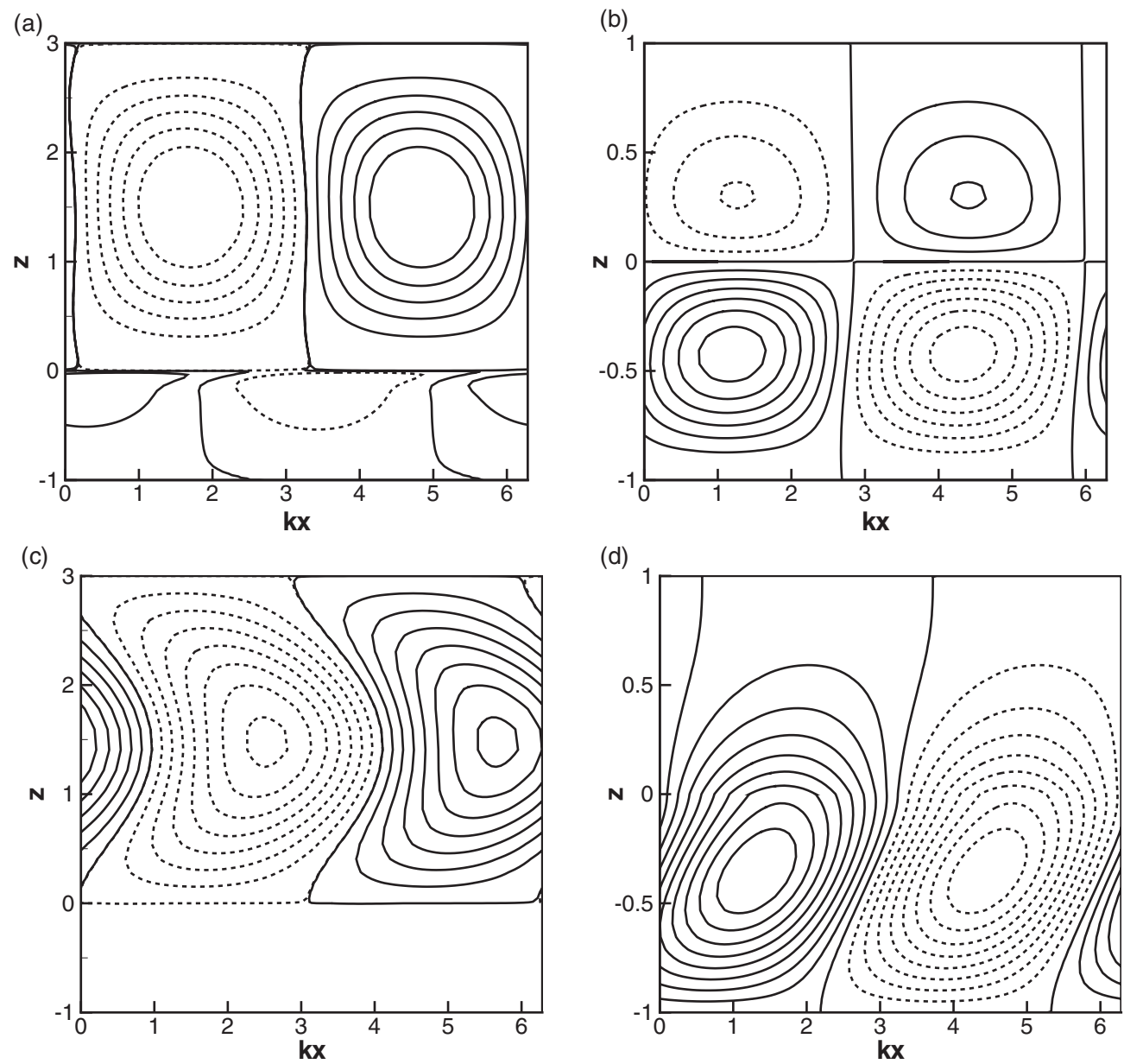

FIG. 3. The flow fields of the stream function of disturbance initiating in (a) the vapor layer and (b) the liquid layer. (c) and (d) are the isothermal lines for temperature disturbance. The parameters for (a) and (c) are $\operatorname{Ra}=1421.8, k=1.04, d=3, K=1$, and $\operatorname{Re}=100$. The parameters for (b) and (d) are $\mathrm{Ra}=984.1, k=2.18, d=1, K=1$, and $\mathrm{Re}=10$.

$$
\lambda U_{v}+i k \bar{u}_{v} U_{v}+D \bar{u}_{v} W_{v}=-\frac{i k}{\rho^{*}} P_{v}+v^{*}\left(D^{2}-k^{2}\right) U_{v}
$$

$$
\lambda W_{v}+i k \bar{u}_{v} W_{v}=-\frac{1}{\rho^{*}} D P_{v}+\frac{\operatorname{Ra} \beta^{*}}{\operatorname{Pr}} \Theta_{v}+v^{*}\left(D^{2}-k^{2}\right) W_{v},
$$

$$
\lambda \Theta_{v}+i k \bar{u}_{v} \Theta_{v}+D \bar{\theta}_{v} W_{v}=\operatorname{Pr}^{-1} \kappa^{*}\left(D^{2}-k^{2}\right) \Theta_{v} .
$$

And for the liquid phase,

$$
\begin{gathered}
i k U_{l}+D W_{l}=0, \\
\lambda U_{l}+i k \bar{u}_{l} U_{l}+D \bar{u}_{l} W_{l}=-i k P_{l}+\left(D^{2}-k^{2}\right) U_{l}, \\
\lambda W_{l}+i k \bar{u}_{l} W_{l}=-D P_{l}+\frac{\operatorname{Ra}}{\operatorname{Pr}} \Theta_{l}+\left(D^{2}-k^{2}\right) W_{l}, \\
\lambda \Theta_{l}+i k \bar{u}_{l} \Theta_{l}+D \bar{\theta}_{l} W_{l}=\operatorname{Pr}^{-1}\left(D^{2}-k^{2}\right) \Theta_{l} .
\end{gathered}
$$

The boundary conditions at the upper wall $z=d$,

$$
U_{v}=W_{v}=\Theta_{v}=0
$$

The boundary conditions at the lower wall $z=-1$,

$$
U_{l}=W_{l}=\Theta_{l}=0 .
$$

The boundary conditions at the initial position of the interface $z=0$ are as follows:

$$
\begin{gathered}
E J=\rho^{*}\left(W_{v}-W_{\mathrm{int}}\right)=W_{l}-W_{\mathrm{int}}, \\
W_{\mathrm{int}}=\lambda h, \\
U_{l}+\frac{\partial \bar{u}_{l}}{\partial z} h=U_{v}+\frac{\partial \bar{u}_{v}}{\partial z} h,
\end{gathered}
$$

$P_{l}-P_{v}-2 D W_{l}+2 \mu^{*} D W_{v}=\left[S k^{2}+\left(1-\rho^{*}\right) G\right] h$,

$$
\left(D U_{l}+i k W_{l}\right)-\mu^{*}\left(D U_{v}+i k W_{v}\right)=-\frac{i k \mathrm{Ma}}{\operatorname{Pr}}\left(\Theta_{l}+D \bar{\theta}_{l} h\right),
$$

$$
\begin{gathered}
J+D \Theta_{l}-\chi^{*} D \Theta_{v}=0, \\
\Theta_{l}+\frac{\partial \bar{T}_{l}}{\partial z} h=\Theta_{v}+\frac{\partial \bar{T}_{v}}{\partial z} h, \\
J=K\left(\Theta_{l}-\frac{1}{\rho^{*}} \frac{\Pi}{E} p_{v}\right) .
\end{gathered}
$$


(a)

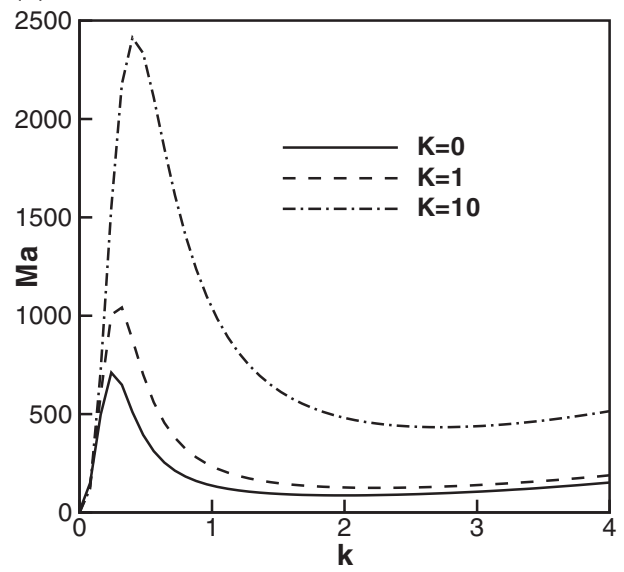

(b)

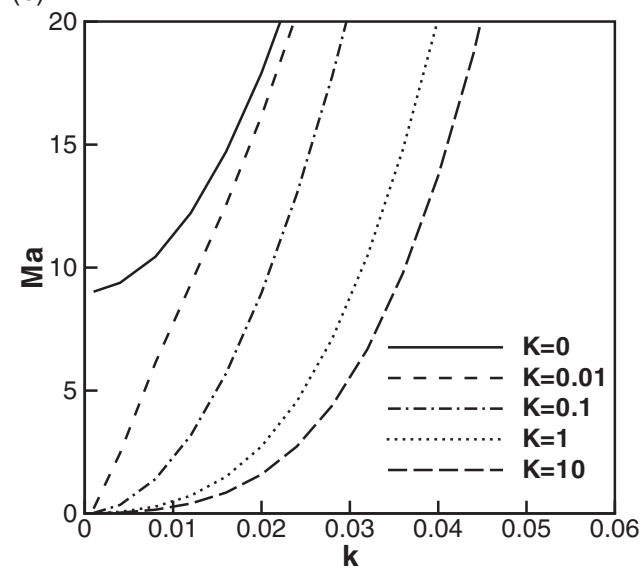

FIG. 4. (a) Effect of $K$ on the marginal curves of the critical Marangoni number versus the wave number at $d=1$, Re $=0$. (b) Effect of $K$ on the marginal curves of the critical Marangoni number versus the wave number in the long-wave range at $d=1, \operatorname{Re}=0$.

Equations (51)-(58) together with the boundary conditions (59)-(68) determine an eigenvalue problem. The goal of the present paper is to determine the conditions at the onset of instability.

\section{NUMERICAL METHOD}

The spectral method can yield great accuracy for the instability problems in hydrodynamics. We implement a Chebyshev collocation method to solve the eigenvalue problem. We first transform the domains of vapor layer $(0, d)$ and liquid layer $(0,-1)$ to the Chebyshev domain $(-1,1)$ by introducing $\eta=$ $2 z / d-1$ and $\eta=2 z+1$. The variables $U_{i}, W_{i}, P_{i}(i=l, v)$ are expanded by Chebyshev series as

$$
\begin{aligned}
U_{i} & =\sum_{j=0}^{N} \hat{U}_{i}^{j} T_{j}(\eta), \quad V_{i}=\sum_{j=0}^{N} \hat{V}_{i}^{j} T_{j}(\eta), \\
W_{i} & =\sum_{j=0}^{N} \hat{W}_{i}^{j} T_{j}(\eta) .
\end{aligned}
$$

Using the Chebyshev series (69), the system of equations is required to solve for $6 N+7$ unknowns including $6 N+6$ spectral coefficients and an interface deflection variable $h$. This gives rise to a $(6 N+7) \times(6 N+7)$ matrix eigenvalue problem in the form of

$$
A X=\lambda B X .
$$

In the present problem, the real part of the complex eigenvalue $\lambda$ is the time growth rate, and the imaginary part is the oscillatory frequency.

\section{RESULTS AND DISCUSSIONS}

The goal of this paper is to investigate the influences of interfacial shear and evaporation on the stability of the system. We chose the system consisting of a water layer in contact with its own vapor at $100^{\circ} \mathrm{C}$. The physical properties of the liquid and the vapor phases are the same with that in Ref. [7]. The ratios of physical properties are $\rho^{*}=6.25 \times$
$10^{-4}, v^{*}=71.72, \chi^{*}=3.68 \times 10^{-2}$, and $\kappa^{*}=0.118$. The Prandtl number is $\operatorname{Pr}=1.78$.

The physics of the coupling of evaporation, interfacial shear, Rayleigh effect, and Marangoni effect is complicated. When investigating the problem, we will look at different aspects of the problem separately whenever possible.

\section{A. Rayleigh-Bénard instability}

We begin with the influence of evaporation and interfacial shear on the Rayleigh convection in the system. The depth ratio is an important parameter to influence the Rayleigh instability of the system. The marginal curves of the critical Rayleigh number versus the wave number are shown in Fig. 2. In these figures, the curves may display a bimodal structure. The left branch corresponds to the mode of convection initiating in the vapor layer, and the right branch corresponds to the convection initiating in the liquid layer. Figure 2(a) shows the effect of the depth ratio on the Rayleigh instability in a system without evaporation. It is observed that, for a smaller depth ratio of $d=2$, the convection initiating in the liquid layer is the dominant mode. With the increase in $d$, the Rayleigh convection in the vapor layer obviously becomes more unstable. However, the increase in $d$ has almost no influence on the Rayleigh convection in the liquid layer (see Ref. [21]). In Fig. 2(a), as $d$ increases to 3, the minimum of the left branch gets lower than that of the right branch. As $d$ increases further, the Rayleigh convection in the vapor layer becomes the dominant mode.

In the present paper, we have used the Hertz-Knudsen equation to predict the evaporation flux. In the dimensionless Hertz-Knudsen equation, parameter $K$ measures the degree of nonequilibrium at the evaporating interface [5]. $K^{-1}=0$ corresponds to the quasiequilibrium case in which the pressure of the vapor is identical to the saturation pressure at the interface; $K=0$ corresponds to the nonvolatile case in which the evaporation flux $J$ is zero. In order to know the effect of the degree of interfacial nonequilibrium on the stability of the system, we plot the marginal curves of the Rayleigh number versus the wave number for various $K$ 's at $d=3$ in Fig. 2(b). 

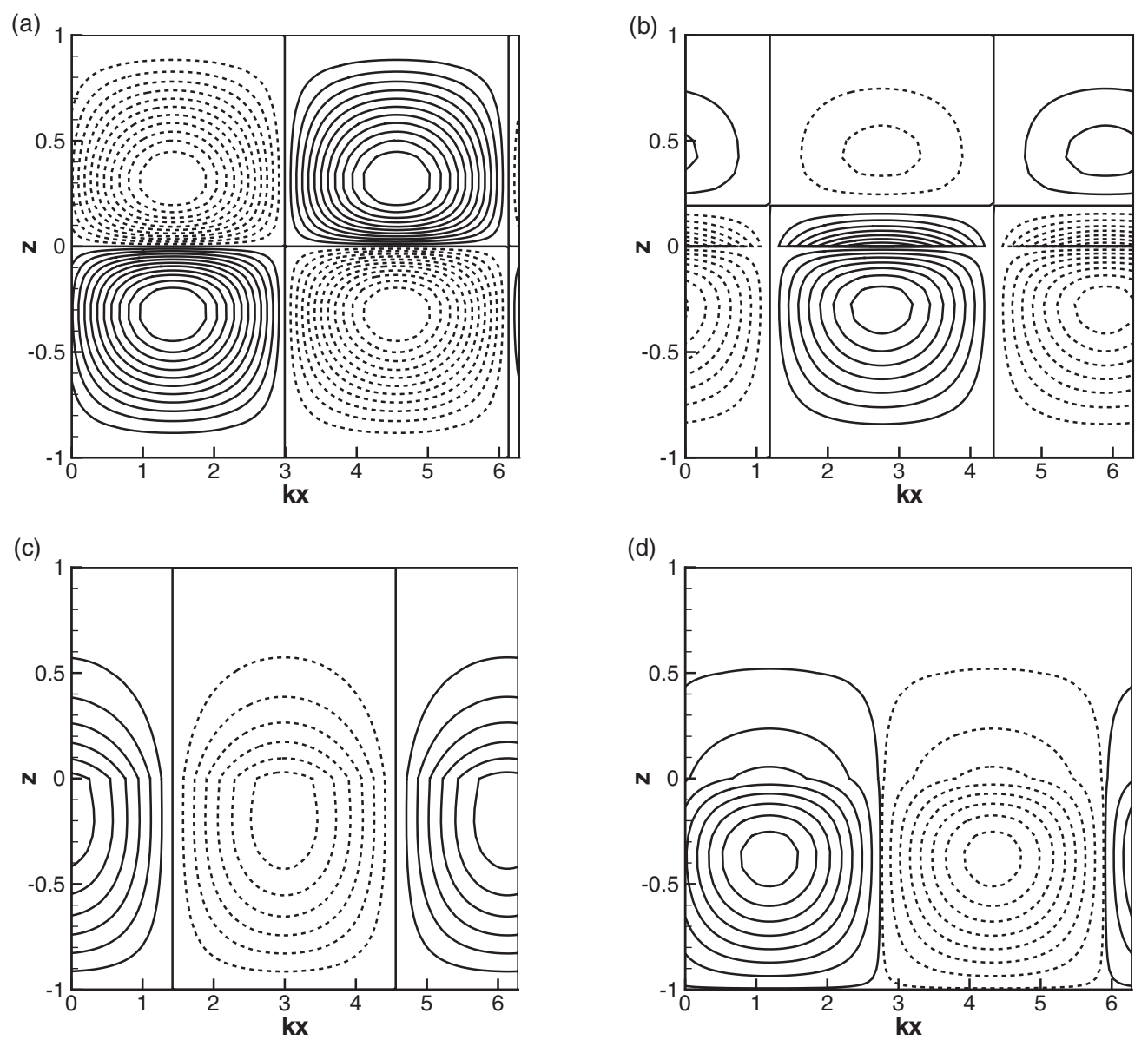

FIG. 5. (a) and (b) The flow fields of the stream function of disturbance. (c) and (d) The isothermal lines for temperature disturbance. The parameters for (a) and (c) are $\mathrm{Ma}=86.9, k=2.0, d=1, K=0$, and $\mathrm{Re}=0$; the parameters for (b) and (d) are $\mathrm{Ma}=433.5, k=2.72, d=$ $1, K=10$, and $\operatorname{Re}=0$.

In this figure, it is shown that, with the increase in $K$, the right branch significantly becomes more stable, however, the left branch is almost unchanged. This result indicates that the degree of equilibrium at the interface plays an important role in the stability of the liquid mode, however, it has almost no influence on the vapor mode.

In order to know the influence of interfacial shear on the stability of the system, we fix other parameters and plot the marginal curves of the Rayleigh number versus the wave number at various Reynolds numbers. As shown in Fig. 2(c), a small change in Re significantly increases the stability of the liquid mode. However, for small Reynolds numbers, the increase in Re only slightly stabilizes the vapor mode. As the Reynolds numbers increase to large values, the vapor mode is the dominant mode, and the increase in Re stabilizes the vapor mode.

In order to know more about the characteristics of the Rayleigh convection of the liquid mode and the vapor mode, we plot the flow fields of these two modes at the onset of convection in Fig. 3. In Fig. 3(a), the Rayleigh convection initiates in the vapor layer, and the liquid layer is moved by the viscous drag of the vapor layer. In this viscous coupling mode of convection, the amplitude of velocity in the liquid layer is much lower than that in the vapor layer. In Fig. 3(b), the convection initiates in the liquid layer, and the vapor layer is driven by the viscous force at the interface. In this case, being different from that in Fig. 3(a), the amplitudes of velocity in both layers are comparable. The isolines of temperature disturbance are plotted in Figs. 3(c) and 3(d) for convection initiating in the vapor layer and the liquid layer. For the vapor mode as shown in Fig. 3(c), the temperature disturbance is mainly confined in the vapor layer. For the liquid mode in Fig. 3(d), the maximum temperature disturbance is located in the liquid layer, and the isolines of the temperature disturbance penetrate deeply into the vapor layer.

\section{B. Marangoni-Bénard instability}

In this subsection, we study the influence of evaporating and interfacial shear on the Marangoni instability. As discussed in Ref. [6], in addition to the classic Marangoni-Bénard mode [22-24], a long-wave Marangoni instability may occur upon the onset of convection. Figure 4 shows the effect of nonequilibrium degree at the interface on the Marangoni instability of the system. In Fig. 4(a), the curves for all $K$ 's display a bimodal structure. The left branch corresponds to the long-wave Marangoni mode, and the right branch corresponds to the classical short-wave Marangoni mode. In this figure, with the increase in $K$, the short-wave mode becomes more stable. In order to know the effect of $K$ on the long-wave 
(a)

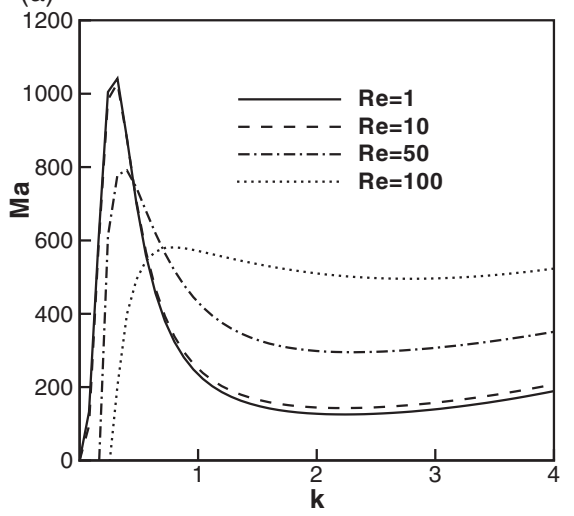

(b)

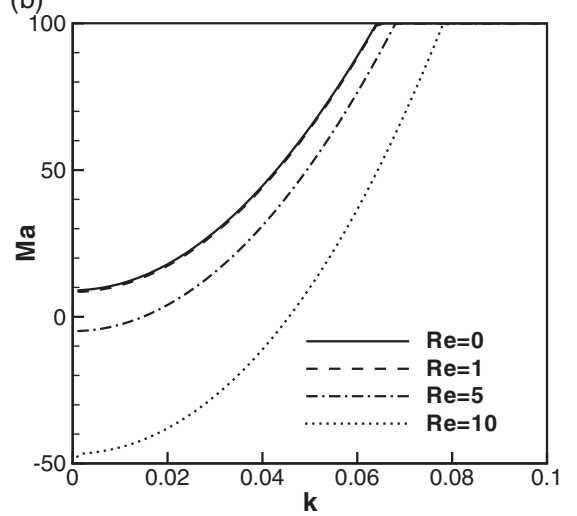

(c)

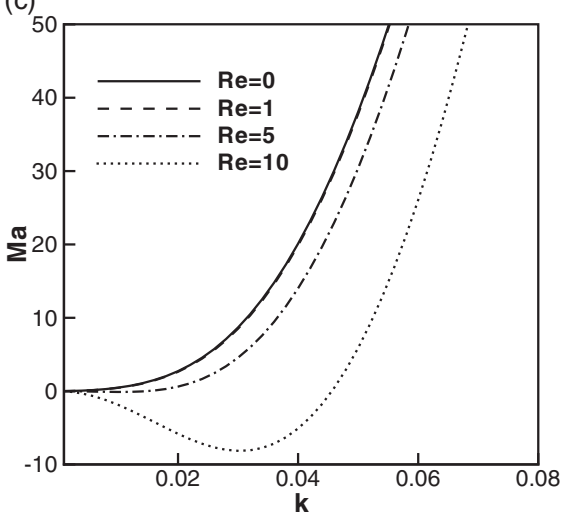

FIG. 6. (a) Effect of Re on the marginal curves of the critical Marangoni number versus the wave number at $d=1, K=1$. (b) Effect of Re on the marginal curves of the critical Marangoni number versus the wave number in the long-wave range at $d=1, K=0$. (c) Effect of Re on the marginal curves of the critical Marangoni number versus the wave number in the long-wave range at $d=1, K=1$.

mode, we plot the closeup of the marginal curves in the longwave range in Fig. 4(b). In the case of $K=0$, the critical condition is realized at $\mathrm{Ma} \simeq 9.0$ and $k=0$. The critical Marangoni number is very sensitive to the change in $K$. As $K$ increases to 0.01 , the critical Marangoni number decreases significantly in the long-wave range and approaches zero as $k \rightarrow 0$. As $K$ increases further, the critical Marangoni number decreases for nonzero wave numbers. However, the increase in $K$ has no influence on the critical Marangoni at the long-wave limit of $k=0$. The curves in Fig. 4 show that the effect of the evaporation is stabilizing for the classical short-wave Marangoni mode and destabilizing for the longwave Marangoni mode. In volatile cases, even when the liquid is not heated from below, the perturbation of evaporation is enough to induce a long-wave Marangoni instability.

In order to know the influence of the evaporation effect on the coupling mode between the vapor and the liquid layers, we plot the flow patterns on the onset of short-wave Marangoni instability in Fig. 5. As shown in Fig. 5(a) for the nonevaporating case, Marangoni convection initiates in the liquid layer, and the circulation in the vapor layer is driven by the viscous force at the interface. The flow pattern is in the form of counter-rotating rolls in the vapor and the liquid layers. However, for the evaporating case, the structure of flow pattern is different from the nonevaporation case. As shown in Fig. 5(b), it is found that, near the interface, the isoline of the stream function extends from the liquid layer to the vapor layer. In this case, the magnitude of velocity in the vapor layer is much lower than that in the liquid layer. This result means that the evaporation at the interface significantly suppresses the viscous coupling between the liquid layer and the vapor layer. The isolines of temperature disturbance are plotted in Figs. 5(c) and 5(d) for the nonevaporating case and the evaporating case. For the nonevaporating case in Fig. 5(c), the maximum temperature disturbance is located near the interface in the liquid layer, and the isolines penetrate into the vapor layer. For the evaporating case in Fig. 5(d), the maximum temperature disturbance is located near the center of the liquid layer. Comparing Fig. 5(d) with Fig. 5(c), in the evaporating case, the temperature disturbance at the interface is much smaller than that in the nonevaporating case. This result means that the evaporation at the interface significantly suppresses the Marangoni effect.

In order to know the effect of interfacial shear on the stability, in Fig. 6, we plot the marginal curves of the critical Marangoni number versus the wave number at various Reynolds numbers. In Fig. 6(a), we observed that, with the increase in the Reynolds number, the curves become (a)

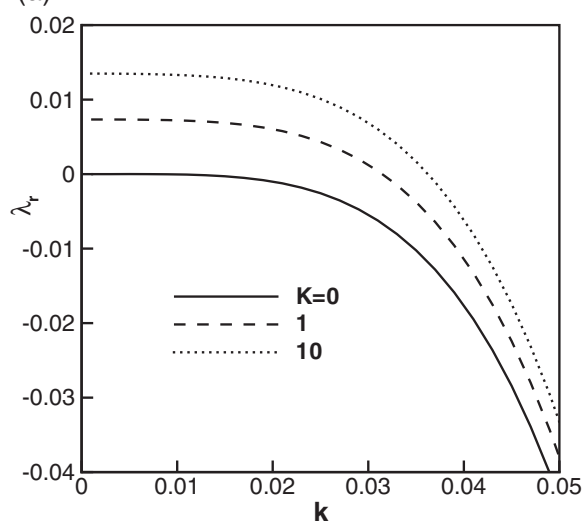

(b)

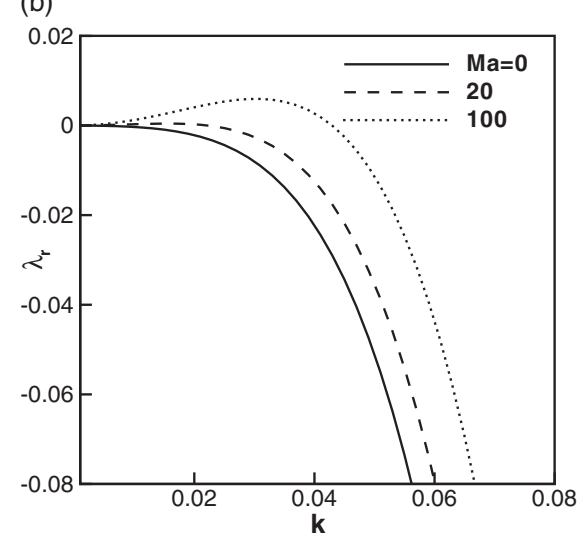

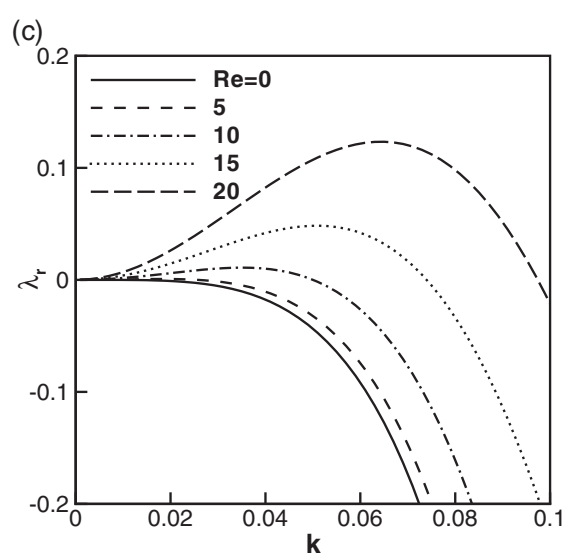

FIG. 7. Dispersion relation between the time growth rate and the wave number. (a) Ma $=10, \operatorname{Re}=0, d=1$ for various $K$, (b) $K=$ $0, \mathrm{Re}=0, d=1$ for various $\mathrm{Ma}$, (c) $K=1, \mathrm{Ma}=1, d=1$ for various $\mathrm{Re}$. 
(a)

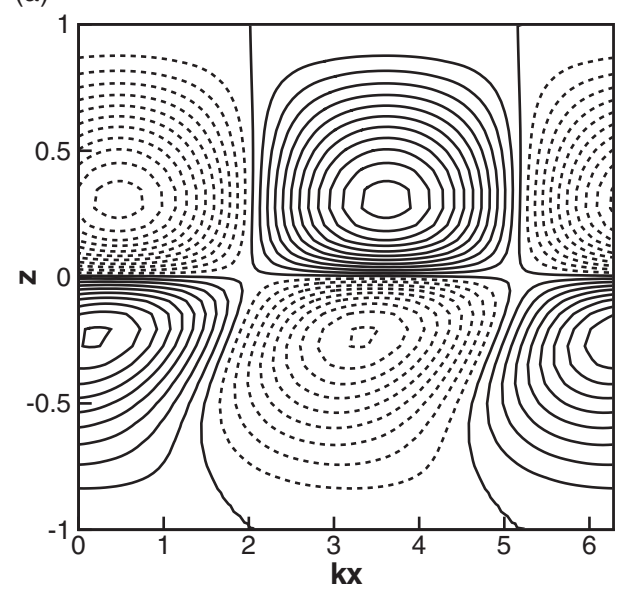

(c)

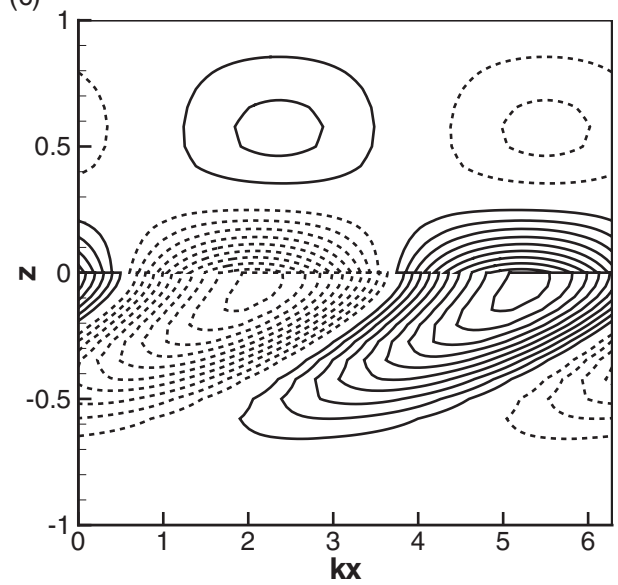

(b)

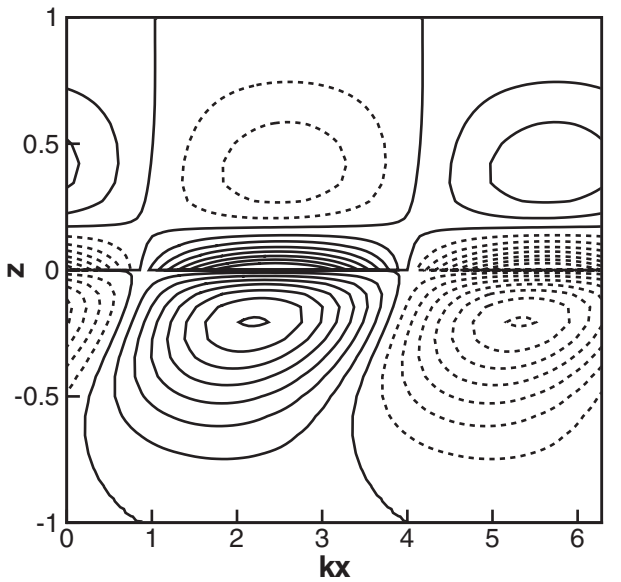

(d)

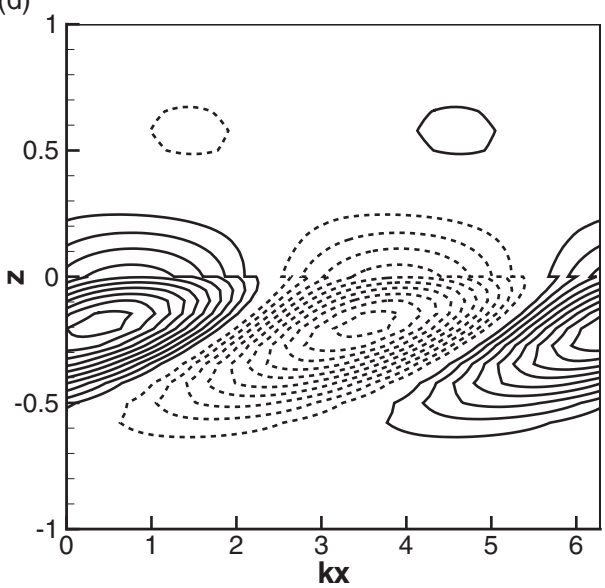

FIG. 8. (a) and (b) The flow fields of stream function of disturbance. (c) and (d) are the isothermal lines for temperature disturbance. The parameters for (a) and (b) are $\mathrm{Ma}=410.5, \alpha=2.44, d=1, K=0$, and $\mathrm{Re}=100$; the parameters for (b) and (d) are Ma $=1159.9, \alpha=$ $3.56, d=1, K=10$, and $\operatorname{Re}=100$.

more stable in the short-wave range and more unstable in the long-wave range. For large values of Re, the long-wave mode is unstable even at a zero Marangoni number. In Figs. 6(b) and 6(c), we present the closeup of the marginal curves for the nonvolatile case of $K=0$ and evaporation case of $K=1$. As shown in Fig. 6(b), for the nonvolatile case, the most unstable long-wave mode is always realized at the longwave limit of $k \rightarrow 0$. In the long-wave range, with the increase in the wave number, the critical Marangoni number increases. However, for the evaporation case of $K=1$ as shown in Fig. 6(c), the critical Marangoni value is always zero at the long-wave limit of $k=0$. For the volatile case of $K=1$, with the increase in $\mathrm{Re}$, the wavelength of the most unstable mode may switch from the long-wave limit to a finite number. With the increase in Re, the long-wave mode becomes more unstable at various wave numbers. In Fig. 6(b), as the Re increases to 10 , the critical Marangoni number has become a negative number. The result in Figs. 6(b) and 6(c) indicates that, in the presence of a cocurrent gas flow, for both the evaporation and the nonvolatile cases, the long-wave Marangoni instability may occur even for a system cooled from below.

The parameters Re, $K$, and Ma play important roles in the long-wave stability of the system. To understand more about the effects of these parameters, it is helpful to look at the dispersion relation between the time growth rate and the wave number. In Fig. 7(a), we present the curves of the dispersion relation for various $K$ 's at $\mathrm{Ma}=10$ and $\mathrm{Re}=0$. In this figure, for all values of $K$, the maximal time growth rate is always realized at $k=0$. With the increase in $K$, the time growth rate increases at all wave numbers in the long-wave range, and Fig. 7(b) presents the influence of Ma on the time growth rate at $\mathrm{Re}=0, K=0$. With the increase in Ma, the time growth rate increases at almost all wave numbers except for $k=0$ in the long-wave range. Moreover, the most amplified disturbance is shifted from the long-wave limit to a finite nonzero wave number. Figure 7(c) shows the effect of interfacial shear on the time growth rate in the long-wave range. It is obvious that, with the increase in the Reynolds number, the time growth rate increases at all wave numbers, and the wave number corresponding to the maximal time growth rate increases.

In Fig. 5, we have studied the influence of evaporation on the coupling mode between the vapor and the liquid layers for evaporation case at $\mathrm{Re}=0$. However, the influence of interfacial shear on the structure of flow field at the onset of short-wave Marangoni instability is unknown. In Fig. 8, the isolines of the stream function and the temperature of 
disturbances are plotted for two typical cases with $\operatorname{Re}>0$. For the nonvolatile case of $K=0$, as shown in Fig. 8(a), the flow pattern consists of counter-rotating rolls in both layers. Being different from the case with $\mathrm{Re}=0$ in Fig. 5, the flow pattern in the liquid layer is in the form of traveling waves and tilts toward the streamwise direction. In Fig. 8(b), for $K=1$, the flow pattern in the liquid layer is qualitatively similar to the nonvolatile case in Fig. 8(a). In the vapor layer, being similar to the case $\mathrm{Re}=0$ as shown in Fig. 5(b), the magnitude of velocity is much smaller than that in the liquid layer because of the evaporation circulation at the interface. Comparing the flow pattern in the vapor and the liquid layers, it is shown that the flow pattern only slightly tilts toward the streamwise direction. In Figs. 8(c) and 8(d), the isolines of the temperature disturbance tilt toward the streamwise direction. For the nonevaporation case in Fig. 8(c), the maximum temperature disturbance is located near the interface in the liquid layer. However, for the evaporation case in Fig. 8(d), the maximum temperature disturbance is located closer to the center of the liquid layer. Comparing Fig. 8(c) with Fig. 8(d), it is shown that, in the evaporating case, the temperature disturbance in the vapor layer is smaller than that in the nonevaporating case.

\section{CONCLUSIONS}

In the present paper, we have studied the instability of a horizontal liquid layer in cocurrent gas flow with an evaporating interface. In this system, the interfacial shear is induced by the cocurrent gas flow. We focus on the effects of evaporation and the interfacial shear on the Rayleigh and Marangoni instabilities.

For the Rayleigh problem, convection can initiate in the vapor layer or in the liquid layer, depending on the depth ratio $d$. The results show that the change in $d$ only influences the vapor mode, and the change in parameter $K$, which represents the nonequilibrium degree at the interface, only influences the critical Rayleigh number of the liquid mode.

Both the evaporation and the interfacial shear play important roles in the Marangoni instability. Parameter $K$ has different influences on the long-wave mode and the short-wave mode of Marangoni instability. With the increase in $K$, the short-wave mode becomes more stable, and the long-wave mode becomes more unstable. The influence of the interfacial shear also has different effects on the short-wave and the long-wave Marangoni instability. With the increase in Re, the short-wave Marangoni mode becomes more stable, and the long-wave mode becomes more unstable.

\section{ACKNOWLEDGMENTS}

The authors gratefully acknowledge support of this work by the European Space Agency (HEAT TRANSFER PRODEX Programme, Belgium). R.L. acknowledges support by the Belgian Federal Science Policy Office.
[1] H. Bénard, Rev. Gen. Sci. Pure Appl. 11, 1261 (1900).

[2] C. A. Miller, AIChE 19, 909 (1973).

[3] A. Prosperetti and M. S. Plesset, Phys. Fluids 27, 1590 (1984).

[4] H. J. Palmer, J. Fluid Mech. 75, 487 (1976).

[5] J. P. Burelbach, S. G. Bankoff, and S. H. Davis, J. Fluid Mech. 195, 463 (1988).

[6] S. J. VanHook, M. F. Schatz, J. B. Swift, W. D. McCormick, and H. L. Swinney, J. Fluid Mech. 345, 45 (1997).

[7] O. Ozen and R. Narayanan, Phys. Fluids , 16, 4644 (2004).

[8] R. Liu and Q. S. Liu, Microgravity Sci. Technol. 21, 233 (2009).

[9] P. Colinet, J. C. Legros, and M. G. Velarde, Nonlinear Dynamics of Surface-Tension-Driven Instabilities (Wiley-VCH, Berlin, 2001).

[10] K. Kanatani, Phys. Fluids 22, 012101 (2010).

[11] K. Kanatani and A. Oron, Phys. Fluids 23, 032102 (2011).

[12] J. Margerit, P. Colinet, G. Lebon, C. S. Iorio, and J. C. Legros, Phys. Rev. E 68, 041601 (2003).
[13] E. Fried, A. Q. Shen, and M. E. Gurtin, Phys. Rev. E 73, 061601 (2006).

[14] D. M. Anderson, P. Cermelli, E. Fried, M. E. Gurtin, and G. B. Mcfadden, J. Fluid Mech. 581, 323 (2007).

[15] O. E. Shklyaev and E. Fried, J. Fluid Mech. 584, 157 (2007).

[16] R. Mahajan, C. Chiu, and G. Chrysler, Proc. IEEE 94, 1476 (2006).

[17] O. A. Kabov, Int. J. Emerging Multidisciplinary Fluid Sci. 2, 87 (2010).

[18] E. Y. Gatapova and O. A. Kabov, Int. J. Heat Mass Transf. 51, 4797 (2008).

[19] C. S. Iorio, O. Goncharova, and O. A. Kabov, Microgravity Sci. Technol. 21, S313 (2009).

[20] O. A. Kabov, D. V. Zaitsev, V. V. Cheverda, and A. B. Cohen, Exp. Therm. Fluid Sci. 35, 825 (2011).

[21] L. Rayleigh, Philos. Mag. 32, 529 (1916).

[22] M. J. Block, Nature (London) 178, 650 (1956).

[23] J. R. A. Pearson, J. Fluid Mech. 4, 489 (1958).

[24] D. A. Nield, J. Fluid Mech. 19, 341 (1964). 